\title{
High resolution measurements of close binary stars and the Probability Imaging technique
}

\author{
Marcel Carbillet, Claude Aime, Eric Aristidi, and Gilbert Ricort \\ Département d'Astrophysique de 1'Université de Nice-Sophia Antipolis \\ URA 709 du CNRS, Parc Valrose, F-06108 Nice cedex 02, France
}

\begin{abstract}
We report in this communication the first experimental results in the visible range obtained by the Probability Imaging technique applied to close binary stars. Speckle data of the well known binary $\beta$ Del as well as the newly discovered one Moai 1 (Carbillet et al. 1996) are processed. An analysis of the probability density functions, combined with the classical visibility, is used to reconstruct the binary systems.
\end{abstract}

\section{Introduction}

An alternative to the computation of moments usualy done in the different speckle interferometry techniques is the analysis of the probability density functions (pdf) at several points in space in the speckle pattern. This is what Probability Imaging (PI) does - see Aime (1993). The two-fold pdf $P^{(2)}\left(\Omega_{1}, \Omega_{2} ; \boldsymbol{\rho}\right)$ is a function of two random variables of intensity $\Omega_{1}$ and $\Omega_{2}$, and a space-lag $\rho$. Whatever the value of $\rho$, the two-fold pdf for a point source has a symmetrical structure in $\Omega_{1}$ and $\Omega_{2}$; when for $\boldsymbol{\rho}$ close to the separation vector $\mathbf{d}$, the two-fold pdf for a binary star has an arrow-head shape with a trend towards a direction $\Omega_{2}$ of the order of $\alpha \Omega_{1}$, where $\alpha$ is the oriented intensity ratio between the two components of the binary system, with no ambiguity about their relative position.

\section{Gaussian Model}

Assuming that the complex amplitude of the wave at the focus of a large telescope is a circular Gaussian process, the two-fold pdf of a binary star can be expressed as the product between the two-fold pdf of a point source and a function $Q\left(\Omega_{1}, \Omega_{2}\right)$ containing all the information about $\alpha$. The value of $\alpha$ can be then easily retrieved doing a simple radial integration of $Q$.

\section{Application}

In practice, the $Q$ function is obtained by dividing the two-fold pdf of the binary star by the point-source one. If the oriented intensity ratio $\alpha$ is not close to 1 , one can compute $Q-Q^{T}$, where $Q^{T}$ is the transpose quantity of $Q$. The radial integration gives then $\alpha$ as well as $\frac{1}{\alpha}$. Otherwise, if $\alpha$ is close to 1 , one only has to compute the radial integration of $Q$, since $Q-Q^{T}$ becomes irrelevant. 
The data processed here were obtained in the red $(\lambda=650 \mathrm{~nm})$ using a specklegraph developed by the Aperture Synthesis group of the Observatoire MidiPyrénées, and coupled with an intensified CCD video camera and an acquisition system. This instrumentation was set at the Cassegrain focus of the $2 \mathrm{~m}$ Bernard Lyot telescope of the Pic du Midi, France. The complete procedure to obtain the relevant parameters $d, \alpha$ and $P A$ (position angle) uses both the classical visibility function and the Q function, as shown in Figure 1.

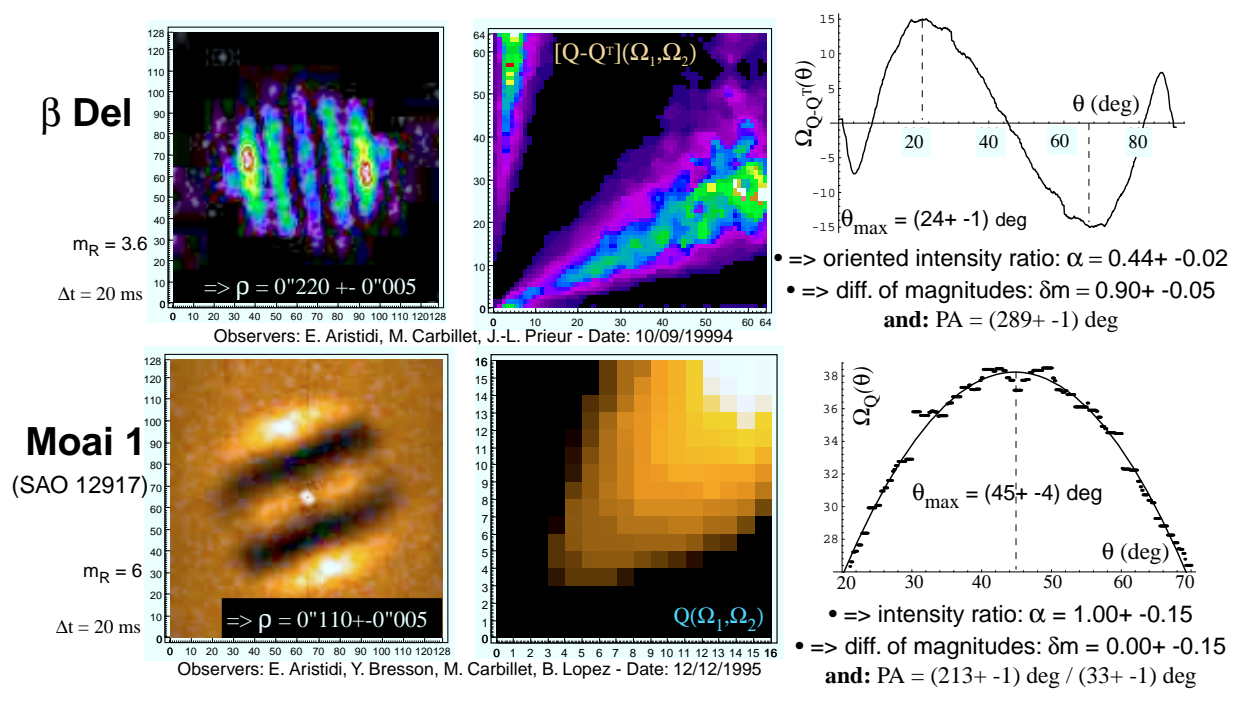

Fig. 1. Square visibility function, $Q-Q^{T}$ (top) or $Q$ (bottom) function and the radial integration of it, for $\beta \operatorname{Del}$ (top) and Moai 1 (bottom).

\section{Perspectives}

The possibility to avoid the use of a reference star is under examination, using the two-fold pdf of the double star computed for $\rho \perp \mathbf{d}$. We also plan to apply this technique as a routine for our current speckle observations, and especially for Post-Hipparcos late-type binary star ones - see Aristidi et al. (1996).

\section{References}

Aime C. (1993): Trends in Opt. Eng.1, 15

Aristidi E., Lopez B., Carbillet M., et al. (1996): this conference

Carbillet M., Lopez B., Aristidi E. et al. (1996): to appear in Astron. Astrophys.

All references available on http://boulega.unice.fr/imager/g2-pub.html 